\title{
Comparison of two path restoration schemes in self-healing networks is
}

\author{
Yijun Xiong ${ }^{\mathrm{a}, *}$, Lorne Mason ${ }^{\mathrm{b}}$ \\ ${ }^{a}$ Department of Electrical and Computer Engineering, Louisiana State University, Baton Rouge, LA 70803, USA \\ b INRS-Telecommunications, 16 Place du Commerce, Verdun, Que., Canada H3E 1 H6
}

Received 3 March 2000; received in revised form 9 May 2001; accepted 11 June 2001

Responsible Editor: K. Shin

\begin{abstract}
The state-independent (SI) and state-dependent (SD) path restoration schemes for self-healing networks are studied in this paper. The integer programming (IP) formulations for the spare capacity allocation and restoration flow assignment are first presented. Based on the optimal IP solutions, the SI and SD restoration schemes are then compared quantitatively in terms of spare capacity requirement (SCR), restoration time, VPI (virtual path identifier) redundancy (in ATM) and nodal storage capacity requirement. The comparison is based on spare optimization, single link failure scenario and $100 \%$ restoration. The SCR is also compared under single node failure scenario. It is observed that using group VP (virtual path) restoration (for either SI or SD) can substantially reduce the restoration time with only a small increase in spare capacity cost. Further, the SI restoration seems better than the SD restoration for the cases we have studied, at least in spare optimization, as its implementation is relatively easier and the difference in SCR between SI and SD is not significant. The economies of scale in transmission facility cost is also investigated in the paper. (C) 2001 Elsevier Science B.V. All rights reserved.
\end{abstract}

Keywords: Network survivability; Path restoration; ATM; Integer programming

\footnotetext{
The research was supported by Louisiana Board of Regents (contract number: LEQSF(1998-01)-RD-A-07) and by a grant from the Canadian Institute for Telecommunications Research under the NCE program of the Government of Canada. Parts of the paper were presented at IEEE ICC'98 (Atlanta, 7-11 June 1998) and at IEEE ATM'98 Workshop (Fairfax, VA, 2629 May 1998).

* Corresponding author. Address: Mahi Networks Inc., 3101 President George Bush Hwy, Suite 200, Richardson, TX 75082, USA.

E-mail address: yxiong@mahinetworks.com (Y. Xiong).
}

\section{Introduction}

Spare capacity requirement (SCR) and restoration time are two important criteria in the design of restorable mesh-type networks. The objective is to provide fast restoration upon network failure at minimal spare cost. Previous study reveals that path restoration usually requires less spare capacity than line (span/link) restoration [1-5]. As the cost of fiber optic transmission systems is still the main cost in the nation-wide transport networks, path restoration appears to be attractive, 
especially in ATM based networks due to the intrinsic features of virtual paths (VPs) [1].

This paper will study two pre-planned path restoration schemes in self-healing networks, with focus on ATM networks. The two restoration schemes are (1) the state-independent (SI) path restoration and (2) the state-dependent (SD) path restoration, which are conceptually illustrated in Fig. 1. In the SI scheme, which is also called the backup VP scheme in ATM [1], each working VP has only one backup VP which takes a link/node disjoint path. When the working VP fails, the affected traffic will be switched to its backup VP. In the SD scheme, each working VP may have more than one "backup VP". The choice of a particular backup VP when the working VP fails will depend on the network failure state. For instance, backup VP 1 will be used when link $(2, D)$ fails and backup VP 2 will be activated when link $(O, 1)$ or $(1,2)$ fails. In both schemes, the spare capacity on each network link is shared among backup VPs of nonsimultaneously failed working VPs. As the SD scheme is tailored to specified failures, it normally requires less spare capacity than the SI scheme.

Given the network topology, traffic (bandwidth) demand matrix and a restoration scheme, one of the basic issues in the design of self-healing ATM networks is how to layout VPs to minimize the spare cost. There are two possible design scenarios: spare optimization (where the layout of working VPs are given) and joint optimization. In either case, the design problem can in general be formulated as integer programming (IP), approximated by linear programming (LP). Here we only consider the spare optimization. The IP formulations for the SI and SD path restorations are first provided, which are solved by using optimization software CPLEX 5.0 [6]. Based on the IP

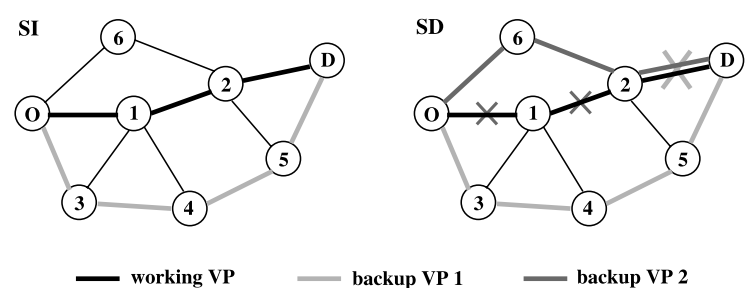

Fig. 1. State-independent (SI) and state-dependent (SD) path restoration schemes. solutions, we then compare these two path restoration schemes quantitatively in terms of SCR, restoration time, VPI (VP identifier) redundancy (in ATM) and node storage capacity. Previous work on this subject can be found in Ref. [7] where the comparisons were based on suboptimal IP solutions. Path elongation factor and routing computational effort were also considered in Ref. [7].

About the capacity allocation and flow assignment problem (VP layout in ATM), a lot more work have been reported in the literature, especially in recently years, for both line and path restoration, e.g., in Refs. [4,5,8-10] and the references cited therein. With regard to path restoration, the SD restoration was studied as an IP in Ref. [4] for both spare and joint optimization. Considering the integrality of physical transmission systems but allowing bifurcated flow restoration, the SI restoration was studied as a mixed IP in Ref. [10] for the spare optimization. The SD restoration was studied as a LP in Refs. [5,11]. The spare cost comparison of the SI and SD restoration was also given in Ref. [5] based on a heuristic spare capacity allocation algorithm. As an extension to the previous work, we consider non-bifurcated VP flow restoration and capacity modularity in physical transmission systems in our IP formulation. The latter allows us to study the economies of scale in transmission facility cost. A restoration protocol which provides an integrated solution to the failure recovery problem in multi-hop networks is described in Ref. [14], where the connection reliability is also evaluated.

The rest of the paper is organized as follows. The IP formulations for the SI and SD restoration are presented in Section 2. Comparisons of the SI and SD restoration schemes on spare capacity cost, VPI redundancy and nodal storage capacity, and restoration time are given in Sections 3-5, respectively. Some concluding remarks are given in Section 6.

\section{Problem formulation}

We use graph $G(\mathscr{N}, \mathscr{L})$ to denote the network under study which has $|\mathscr{N}|$ nodes and $|\mathscr{L}|$ links, where $\mathscr{N}$ and $\mathscr{L}$ are the sets of network nodes and 
links, respectively, and $|\mathscr{N}|$ is the cardinality of set $\mathscr{N}$. Each link $l \in \mathscr{L}$ consists of two arcs ( $a$ and $a^{\prime} \in \mathscr{A}$ ) which have the same end nodes as $l$ but with opposite directions. $\mathscr{A}$ is the set of arcs. Let $\mathscr{S}$ be the set of states of the network and $s_{0}(\in \mathscr{S})$ be the network normal operating state (no failure). For a single link failure scenario, $|\mathscr{S}|=|\mathscr{L}|+1$. Further, let

$\Pi$ : the set of origin-destination node pairs (commodities);

$O(\pi): \quad$ the origin node of commodity $\pi, \pi \in \Pi$;

$D(\pi)$ : the destination node of commodity $\pi$, $\pi \in \Pi$;

$\mathscr{V}_{\pi}$ : the set of VPs of commodity $\pi, \pi \in \Pi$;

$\gamma_{\pi v}$ : the traffic demand expressing bandwidth requirement for VP $v$ of commodity $\pi$, $v \in \mathscr{V}_{\pi}$;

$R_{\pi v}^{s}$ : the set of candidate routes for VP $v$ of commodity $\pi$ in network state $s \in \mathscr{S}$;

$x_{r \pi v}^{s}$ : the normalized bandwidth used by VP $v$ of commodity $\pi$ on route $r$ when the network is in state $s, v \in \mathscr{V}_{\pi}, r \in R_{\pi v}^{s}, \pi \in \Pi$ and $s \in \mathscr{S}$;

$f_{\pi v}^{s}$ : the restoration level, $0 \leqslant f_{\pi v}^{s} \leqslant 1, \pi \in \Pi$, $v \in \mathscr{V}_{\pi}$ and $s \in \mathscr{S}$

$\delta_{r \omega}: \quad$ the delta function which equals 1 when network component $\omega$ is on route $r$ and 0 otherwise;

$\mathscr{F}(s)$ : a set of failed components (links and nodes) when the network is in state $s \in \mathscr{S}-s_{0}$;

$c_{a}^{\text {spare: }} \quad$ spare capacity on arc $a \in \mathscr{A}$;

$c_{a}^{\text {work }}$ : $\quad$ working capacity on arc $a \in \mathscr{A}$;

$d_{a}$ : length of arc $a \in \mathscr{A}$;

$\mathscr{T}: \quad$ the set of candidate optical fiber cable types;

$Z_{a}^{t}$ : number of type $t$ cables used on $\operatorname{arc} a$, $a \in \mathscr{A}, t \in \mathscr{T}$;

$C^{t}$ : cost of a type $t$ cable per unit length, $t \in \mathscr{T}$

$B^{t}: \quad$ capacity (in Mbps) of a type $t$ cable, $t \in \mathscr{T}$.

It is assumed that no statistical multiplexing is allowed between VPs passing the same link and link cost is proportional to the link length. Let us first consider the case of no capacity modularization. In this case the capacity allocation and flow assignment in self-healing networks with the SD path restoration can be formulated as the following mixed IP problem.

F1 Minimize $\sum_{a \in \mathscr{A}} d_{a}\left(c_{a}^{\text {work }}+c_{a}^{\text {spare }}\right)$,

subject to

$$
\begin{aligned}
& \sum_{r \in R_{\pi v}^{s_{0}}} x_{r \pi v}^{s_{0}}=1, \quad v \in \mathscr{V}_{\pi}, \pi \in \Pi, \\
& c_{a}^{\mathrm{work}}=\sum_{\pi \in \Pi} \sum_{v \in \mathscr{V}_{\pi}} \sum_{r \in R_{\pi v}^{s_{0}}} \delta_{r a} \gamma_{\pi v} x_{r \pi v}^{s_{0}}, \quad a \in \mathscr{A}, \\
& \sum_{r \in R_{\pi v}^{s}} x_{r \pi v}^{s}=\sum_{r \in R_{\pi v}^{s_{0}}} \Omega(\pi, r, s) x_{r \pi v}^{s_{0}}, \quad v \in \mathscr{V}_{\pi}, \\
& c_{a}^{\mathrm{spare}} \geqslant \sum_{\pi \in \Pi} \sum_{v \in \mathscr{V}_{\pi}} \sum_{r \in R_{\pi v}^{s}} \delta_{r a} \gamma_{\pi v} f_{\pi v}^{s} x_{r \pi v}^{s}, \quad s \in \mathscr{S}-s_{0}, \\
& \quad a \in \mathscr{A}, \quad s \in \mathscr{S}_{-}, \\
& x_{r \pi v}^{s} \in\{0,1\}, \quad v \in \mathscr{V}_{\pi}, \pi \in \Pi, \quad s \in \mathscr{S}, \quad r \in R_{\pi v}^{s},
\end{aligned}
$$

where

$\Omega(\pi, r, s)= \begin{cases}1, & \text { if } \mathscr{F}(s) \bigcap\{r\} \neq \emptyset \text { and } \\ & \{O(\pi), D(\pi)\} \bigcap \mathscr{F}(s)=\emptyset, \\ 0, & \text { otherwise. }\end{cases}$

In the above, constraints (1) and (2) assure that the bandwidth demand $\gamma_{\pi v}$ of a VP is satisfied. Constraint (3) ensure that a fraction of an affected working VP will be restored. The constraints in Eq. (4) guarantee that sufficient spare capacity is allocated to each arc to meet the restoration requirement. Constraint (5) means that VP flows are non-bifurcated. Throughout the paper we assume $100 \%$ restoration for the predefined failure scenario, i.e., $f_{\pi v}^{s}=1$. Note that formulation F1 is suitable for both spare and joint optimization. For spare optimization, constraints (1) and (2) can be removed since the working VP routes are given and the working capacity $c_{a}^{\text {work }}$ can be calculated from Eq. (2).

When the capacity modularity is considered, the above formulation becomes 
F2 Minimize $\sum_{a \in \mathscr{A}} \sum_{t \in \mathscr{T}} d_{a} Z_{a}^{t} C^{t}$,

subject to Eqs. (1)-(5) and

$\sum_{t \in \mathscr{T}} Z_{a}^{t} B^{t} \geqslant c_{a}^{\text {work }}+c_{a}^{\text {spare }}, \quad a \in \mathscr{A}$,

$Z_{a}^{t} \geqslant 0$ and integer, $a \in \mathscr{A}, t \in \mathscr{T}$.

The IP formulation for the SI path restoration can be obtained in a similar manner. Let us consider only spare optimization here. Denote by $p_{\pi v}^{s_{0}}$ the working path of VP $v$ of commodity $\pi$ and $x_{r \pi v}$ its candidate restoration path. Let $R_{\pi v}$ be the set of restoration routes which are link/node disjoint to $p_{\pi v}^{s_{0}}$. We have the following IP formulation for the SI path restoration (without link modularization):

F3 Minimize $\sum_{a \in \mathscr{A}} d_{a} c_{a}^{\text {spare }}$,

subject to

$\sum_{r \in R_{\pi v}} x_{r \pi v}=1, \quad v \in \mathscr{V}_{\pi}, \quad \pi \in \Pi$,

$c_{a}^{\text {spare }} \geqslant \sum_{\pi \in \Pi} \sum_{v \in \mathscr{V}_{\pi}} \sum_{r \in R_{\pi v}} \delta_{r a} \gamma_{\pi v} f_{\pi v}^{s} \Omega\left(\pi, p_{\pi v}^{s_{0}}, s\right) x_{r \pi v}$,

$$
a \in \mathscr{A}, s \in \mathscr{S}-s_{0},
$$

$x_{r \pi v} \in\{0,1\}, \quad v \in \mathscr{V}_{\pi}, \quad \pi \in \Pi, \quad r \in R_{\pi v}^{s}$.

In the above formulation, constraints (8) and (10) ensure that there is only one backup VP for each working VP. Note that formulation F2 is still valid when taking into account link modularization if replacing constraints from $\mathrm{F} 1$ by the constraints from F3.

In formulation $\mathrm{F} 1$ the total number of constraints $N_{\mathrm{c}}$ is roughly equal to $|\mathscr{S}|\left(\sum_{\pi \in \Pi}\left|\mathscr{V}_{\pi}\right|+\right.$ $|\mathscr{A}|)$, and the total number of variables $N_{\mathrm{v}}$ is about $\sum_{s \in \mathscr{S}} \sum_{\pi \in \Pi} \sum_{v \in \mathscr{V}_{\pi}}\left|R_{\pi v}^{s}\right|$, while in formulation $\mathrm{F} 3, \quad N_{\mathrm{c}} \approx \sum_{\pi \in \Pi}\left|\mathscr{V}_{\pi}^{\pi}\right|+|\mathscr{A}||\mathscr{S}|$ and $N_{\mathrm{v}}=$ $\sum_{\pi \in \Pi} \sum_{v \in \mathscr{V}_{\pi}}\left|R_{\pi v}^{s}\right|$. Clearly, the number of constraints and variables in F3 are much less than those in $\mathrm{F} 1$, roughly only one $|\mathscr{S}|$-th of those in F1. However, in either case (SD or SI), the size of the problem grows rapidly with $\left|\mathscr{V}_{\pi}\right|$, the number of VPs between a node pair. To reduce the problem size and to study large-scale networks, we use the group VP concept in F1 and F3 which treats
VPs between a node pair as one super VP (i.e., $\left.\left|V_{\pi}\right|=1\right)$. And we found that in many cases it can yield near-optimal and sometimes optimal solutions to the original problem.

Note that the above formulations are also applicable for the design of self-healing SONET/ SDH networks by restricting the bandwidth of each VP to OC-n/STM- $m$.

In the following, we first compare the SI and $\mathrm{SD}$ restoration schemes in terms of SCR.

\section{Spare capacity requirement}

\subsection{Network topology and traffic demand}

New Jersey LATA network (LATA_Net) and US long-haul network (US_Net) are selected in our numerical study [5], which are shown in Fig. 2. LATA_Net has 11 nodes and 23 links while US_Net has 28 nodes and 45 links. Here we only consider a subnet of US_Net, denoted by US_Net $(20,30)$, which consists of nodes 0-19 and related links (30 in total) (see Fig. 2(b)). In this paper, we simply use US_Net to indicate US_Net $(20,30)$ unless otherwise mentioned. Both uniform and non-uniform traffic demands are considered. For uniform demand (UD), the traffic demand between each node pair is the same, i.e., $\gamma_{\pi}=\sum_{v \in \mathscr{V}_{\pi}} \gamma_{\pi v}=\eta, \pi \in \Pi$. For non-uniform demand (ND), the traffic demand between a node pair is inversely proportional to the hops of the shortest path between that node pair, i.e., $\gamma_{\pi}=$ $\eta / h(\pi)$. For the node pair $\pi=(O, D)$ in Fig. 1, $h(\pi)=3$. Suppose all working VPs are given which take the shortest paths. The optimal layout of backup VPs which gives the minimal spare cost is obtained by solving formulation F1/F3 (or F2 if link modularization is considered) using the CPLEX 5.0 optimization software package [6]. Denote by $C_{\mathrm{w}}$ the working capacity cost provided that all working VPs take the shortest paths. It is clear that $C_{\mathrm{w}}$ is unique for the given network topology and traffic demand. Note that $C_{\mathrm{w}}$ is not necessary the real working capacity cost. The SCR is defined here as the ratio of the total spare capacity cost (denoted by $C_{\mathrm{s}}$ ) to the total working capacity cost, i.e., 
(a)

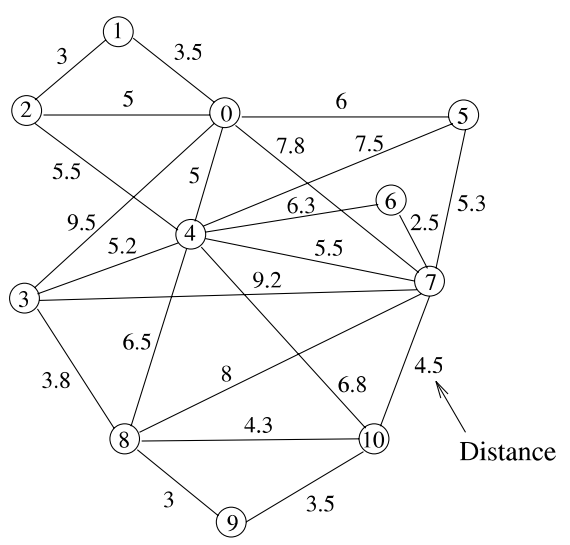

(b)

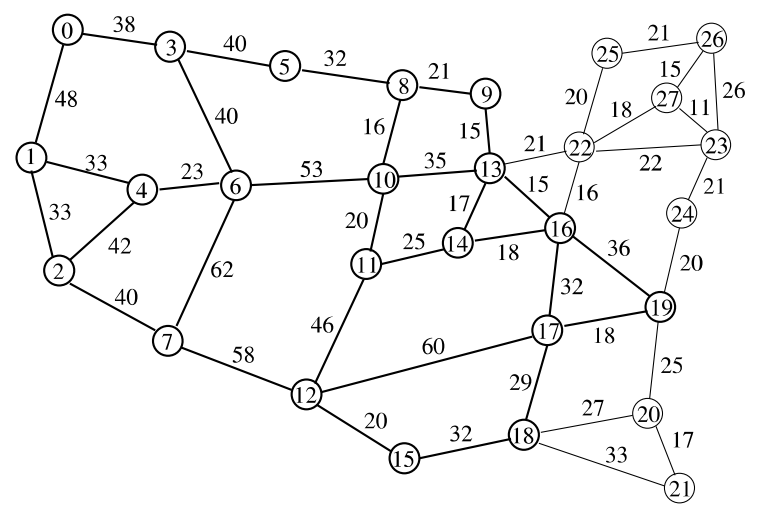

Fig. 2. (a) New Jersey LATA network, (b) US long-haul network.

$\mathrm{SCR}=\frac{C_{\mathrm{s}}}{C_{\mathrm{w}}}=\frac{C_{\mathrm{n}}-C_{\mathrm{w}}}{C_{\mathrm{w}}}$,

where $C_{\mathrm{n}}$ is the total network cost.

Given the traffic demand $\gamma_{\pi}$, there are numerous ways to choose the VP set $\mathscr{V}_{\pi}$ and the bandwidth $\gamma_{\pi v}$ of each VP $v \in \mathscr{V}_{\pi}$. Here we consider two extreme cases (1) each node pair has only one VP, i.e., $\left|\mathscr{V}_{\pi}\right|=1$ and (2) $\gamma_{\pi}$ can be arbitrarily partitioned (in other words, the traffic demand is simply modeled as commodity flow). Obviously, the former will give an upper bound on SCR while the latter will lead to a lower bound on SCR. Formulations F1 and F3 in case (2) are actually re- duced to LP (constraints (5) in F1 and constraints (10) in F3 are relaxed). In practice, there is usually more than one VP per node pair in order to support various traffic classes or different QoS requirements of the same class.

The numerical results given below are obtained from spare optimization only. To reduce the computational complexity, the number of candidate restoration routes $M$ per node pair is limited to 10 . Specifically, mutually link/node disjoint paths plus the shortest paths (not necessary working VP link/node disjoint) are used in the SD scheme and $M$ shortest working VP link/node disjoint paths are used in the SI scheme. As an example, the sets of candidate restoration routes for node pair $(1,8)$ in LATA_Net are given in Table 1 under a single link failure scenario. It is clear that in the SD scheme, $\left|R_{\pi v}^{s}\right| \leqslant M, s \in \mathscr{S}-s_{0}$, because a working VP and some of its candidate restoration routes may pass the same link. In the SI scheme, $\left|R_{\pi v}^{s}\right|=M$. The hop limits in selecting path sets are 6 and 10 respectively, for LATA_Net and US_Net.

Fig. 3 shows the SCR versus the number of candidate routes in US_Net. The SCR is calculated based on the LP solution of F3 (SI restoration). As one can see, SCR becomes less and less sensitive to $M$, the number of candidate routes, as $M$ increases, which is especially true for small networks like LATA_Net.

Table 1

Two sets of candidate restoration routes between nodes 1 and 8 in LATA_Net

\begin{tabular}{llllll}
\hline \multirow{2}{*}{ No. } & \multicolumn{2}{l}{ Scheme } & & & \\
\cline { 2 - 3 } & SD & & SI & \\
\cline { 2 - 3 } \cline { 5 - 6 } & Route & Length & & Route & Length \\
\hline 1 & $1-2-4-3-8$ & 17.5 & & $1-2-4-3-8$ & 17.5 \\
2 & $1-2-4-8$ & 15.0 & & $1-2-4-10-8$ & 19.8 \\
3 & $1-0-3-8$ & 16.8 & & $1-2-0-3-8$ & 21.3 \\
4 & $1-0-4-3-8$ & 17.5 & & $1-2-4-10-9-8$ & 21.8 \\
5 & $1-0-7-8$ & 19.3 & & $1-2-4-7-8$ & 22.0 \\
6 & $1-2-0-4-8$ & 19.5 & & $1-2-4-7-10-8$ & 23.0 \\
7 & $1-0-4-10-8$ & 19.8 & & $1-2-0-7-8$ & 23.8 \\
8 & $1-2-4-10-8$ & 19.8 & & $1-2-0-7-10-8$ & 24.8 \\
9 & $1-0-7-10-8$ & 20.3 & & $1-2-4-7-10-9-8$ & 25.0 \\
10 & $1-0-2-4-8$ & 20.5 & $1-2-4-6-7-8$ & 25.3 \\
\hline
\end{tabular}

Working VP: $1-0-4-8$ (length $=15.0$ ). 


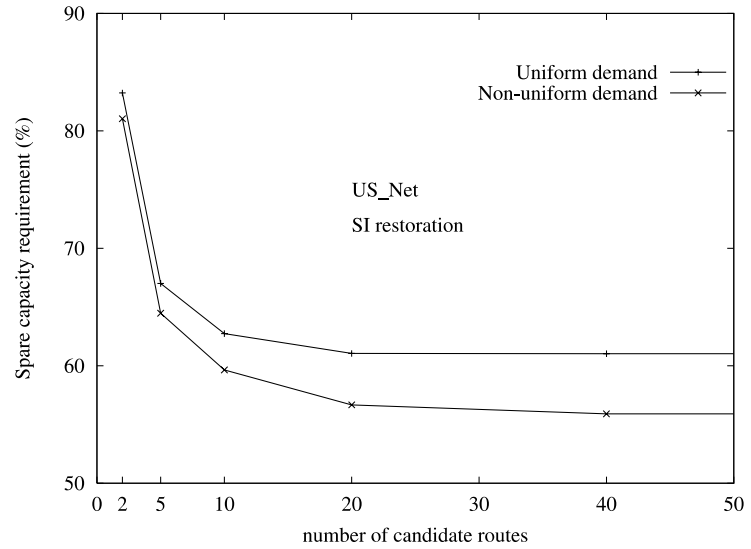

Fig. 3. SCR versus number of candidate routes in US_Net with SI restoration.

\subsection{Spare optimization: LP vs. IP}

Again, let us first consider that there is no capacity modularity (F1/F3). Tables 2 and 3 compare the SCRs under two different failure

Table 2

SCR under state-dependent $(\mathrm{SD})$ path restoration $(M=10)$

\begin{tabular}{|c|c|c|c|c|}
\hline \multirow{3}{*}{$\begin{array}{l}\text { Optimi- } \\
\text { zation }\end{array}$} & \multicolumn{4}{|c|}{ Scenario } \\
\hline & \multicolumn{2}{|c|}{ Link failure } & \multicolumn{2}{|c|}{ Link/node failure } \\
\hline & LP $(\%)$ & IP-LP (\%) & LP $(\%)$ & IP-LP $(\%)$ \\
\hline \multicolumn{5}{|l|}{$L A T A$} \\
\hline UD & 59.11 & 0.14 & 82.16 & 0 \\
\hline ND & 52.52 & 2.16 & 65.72 & 3.00 \\
\hline \multicolumn{5}{|l|}{ US_Net } \\
\hline UD & 66.14 & 0 & 74.76 & 0 \\
\hline ND & 61.58 & 2.84 & 67.02 & 0.85 \\
\hline
\end{tabular}

Table 3

SCR under state-independent $(\mathrm{SI})$ path restoration $(M=10)$

\begin{tabular}{|c|c|c|c|c|}
\hline \multirow{3}{*}{$\begin{array}{l}\text { Optimi- } \\
\text { zation }\end{array}$} & \multicolumn{4}{|c|}{ Scenario } \\
\hline & \multicolumn{2}{|c|}{ Link failure } & \multicolumn{2}{|c|}{ Link/node failure } \\
\hline & LP (\%) & IP-LP (\%) & LP $(\%)$ & IP-LP $(\%)$ \\
\hline \multicolumn{5}{|l|}{$L A T A$} \\
\hline UD & 57.48 & 0 & 73.92 & 0 \\
\hline ND & 51.48 & 1.56 & 62.06 & 1.61 \\
\hline \multicolumn{5}{|l|}{$U S \_N e t$} \\
\hline UD & 61.60 & 0.18 & 67.71 & 0.04 \\
\hline ND & 57.44 & 4.51 & 60.14 & 1.23 \\
\hline
\end{tabular}

scenarios: any single link failure and any single link or node failure. They show that the difference in SCR between IP (case 1) and LP (case 2) is only marginal, no more than $5 \%$ in all our numerical results, for both SI and SD schemes. LP results are obtained by removing constraint (5) in F1 and (10) in F3. This may be due to the fact that arc spare capacity is shared among backup VPs of nonsimultaneously failed working VPs. The spare capacity on each arc is only determined by the worst failure case which requires the highest spare capacity on that arc. The above results indicate that to treat working VPs between a node pair as a group during restoration the penalty in SCR is small. But on the other hand, the restoration speed could be much faster (see Fig. 7) because the number of restoration cells generated at failure event is greatly reduced.

Note that the SCRs under the SD and SI schemes are different because the path sets used in F1 and F3 are different. If the same path set is used, the SCR of the SD scheme should not be larger than that of the SI scheme (see Table 4), as the SD scheme is more flexible and tailored to each failure case. However, we observe from Table 4 that the difference is very small. The reason might be the same as what we have explained for LP versus IP case. If this is true in general, then the SI scheme has advantages over the SD scheme when consider implementation complexity, e.g., nodal storage capacity and VPI (VP identifier) redundancy (to be discussed in the next section), at least for spare optimization.

Table 4

SCRs of SD and SI schemes using the same path set $(M=10)$, IP solution

\begin{tabular}{|c|c|c|c|c|}
\hline \multirow{3}{*}{$\begin{array}{l}\text { Optimi- } \\
\text { zation }\end{array}$} & \multicolumn{4}{|l|}{ Scenario } \\
\hline & \multicolumn{2}{|c|}{ Link failure } & \multicolumn{2}{|c|}{ Link/node failure } \\
\hline & $\mathrm{SD}(\%)$ & SI-SD $(\%)$ & $\mathrm{SD}(\%)$ & SI-SD $(\%)$ \\
\hline \multicolumn{5}{|l|}{$L A T A$} \\
\hline UD & 57.48 & 0 & 73.92 & 0 \\
\hline ND & 53.04 & 0 & 62.82 & 0.85 \\
\hline \multicolumn{5}{|l|}{$U S \_N e t$} \\
\hline UD & 61.72 & 0.06 & 67.75 & 0 \\
\hline ND & 60.64 & 1.31 & 61.37 & 0 \\
\hline
\end{tabular}


Table 5

SCR under single link failure scenario and $(M=40)$

\begin{tabular}{llllll}
\hline \multirow{2}{*}{$\begin{array}{l}\text { Optimi- } \\
\text { zation }\end{array}$} & \multicolumn{2}{l}{ Network } & & \\
\cline { 2 - 3 } \cline { 5 - 5 } & \multicolumn{2}{l}{ LATA_Net } & & US_Net \\
\cline { 2 - 3 } & LP (\%) & IP-LP (\%) & & LP (\%) & IP-LP (\%) \\
\hline SD & & & & \\
UD & 56.46 & 0 & 61.15 & 0.27 \\
ND & 51.30 & 0.42 & 56.89 & 0.91 \\
SI & & & & \\
UD & 56.55 & 0.16 & 61.03 & 0.14 \\
ND & 51.30 & 0.70 & 55.91 & 1.60 \\
\hline
\end{tabular}

Very similar results are observed when $(M=$ 40) (see Table 5), and also when considering capacity modularity (F2). Again the results in Table 5 are obtained when the two restoration schemes use different path sets. As expected, when $M$ increases, the SCR decreases. Moreover, the difference in SCR between two restoration schemes also becomes smaller. As the number of eligible candidate restoration routes in SD restoration is much larger than that in SI restoration, if $M$ is further increased, it is expected that the SCR in the SD case would be smaller than that in the SI case. However, the gap may not be significant since the SCR is not sensitive to $M$ when $M$ is sufficiently large (see Fig. 3). Also, when $M$ is too large, the optimization solution may be infeasible due to huge memory space requirement.

It is worthy to mention that SD restoration is more suitable for joint optimization than SI restoration. For some network topologies and traffic demands, the gap of total network cost between joint and spare optimization could be significant [5]. In such a situation, there would be a trade-off between capacity cost and implementation complexity when choosing an appropriate restoration scheme.

\subsection{Impact of link modularity}

The formula "cost equals bandwidth to power 0.6 " is used to calculate the cable cost for the given cable transmission capacity. Three cable types are used in formulation F2 with capacity $B^{1}=1$, $B^{2}=4, B^{3}=16$ and cost $C^{1}=1, C^{2}=2.297$, $C^{3}=5.278$, respectively. One unit of bandwidth
Table 6

Total network cost with and without capacity modularization

\begin{tabular}{|c|c|c|c|c|c|}
\hline \multicolumn{2}{|c|}{ Formulation } & $\mathrm{F} 1(w / o)$ & $\mathrm{F} 1(w)$ & $\mathrm{F} 2$ & $\Delta(\%)$ \\
\hline \multicolumn{6}{|c|}{ LATA_Net } \\
\hline UD & $\eta=1$ & 784 & 481 & 441 & 9.07 \\
\hline ND & $\eta=6$ & 2648 & 1061 & 987 & 7.50 \\
\hline \multicolumn{6}{|c|}{$U S \_N e t$} \\
\hline \multirow[t]{2}{*}{ UD } & $\eta=1$ & 30,452 & 11,292 & 11,144 & 1.33 \\
\hline & $\eta=0.3$ & 9136 & 4781 & 4620 & 3.48 \\
\hline \multirow[t]{2}{*}{ ND } & $\eta=12$ & 118,080 & 40,503 & 39,863 & 1.61 \\
\hline & $\eta=1$ & 9002 & 4803 & 4585 & 4.76 \\
\hline
\end{tabular}

$\Delta=(\mathrm{F} 1(w)-\mathrm{F} 2) / \mathrm{F} 2$.

could be regarded as 150 Mbps (OC-3/STM-1). The total network costs with and without link modularization are shown in Table 6 . The data in column $\mathrm{F} 1(w)$ are the cost from $\mathrm{F} 1$ by taking into account the modularity (roundup). We observe that the difference between $\mathrm{F} 2$ and $\mathrm{F} 1(w)$ really depends on the traffic demand and it could be significant for some traffic patterns. Needless to say, there will be a big saving by using high capacity fiber cables whenever possible. Because of link modularity, We also expect the conclusions in the previous subsection on LP versus IP and SI versus SD restoration are still valid here.

\section{VPI redundancy and node storage capacity}

\subsection{VPI redundancy}

The working or backup VP route in an ATM network is defined by a sequence of arcs as well as VPIs on these arcs. Using preplanned restoration approach, like the SD or SI scheme, a certain amount of VPIs must be reserved on each arc in order to activate the restoration routes upon network failure. There are two possible ways to assign VPIs to backup routes, (1) to preassign VPIs to backup routes before failure occurs (called VPI preassignment method), (2) to assign VPIs only at failure event to those backup routes that will be activated (termed VPI non-preassignment method). Obviously, the preassignment method will reserve much more VPIs than the non-preassignment method. In this section we try to estimate 
the VPI redundancy for both methods when using the SD and SI restoration schemes.

The VPI redundancy is defined here as the ratio of the total number of VPIs reserved for restoration routes to the total number of VPIs used by working VPs, i.e.,

$\mathrm{VR}=\frac{\sum_{a \in \mathscr{A}} \mathrm{RV}(a)}{\sum_{a \in \mathscr{A}} \mathrm{UV}(a)}$

Denoting by $p_{\pi v}^{s_{0}}$ the working path for VP $v$ of commodity $\pi$, it is clear that the number of used VPIs on arc $a$

$$
\mathrm{UV}(a)=\sum_{\pi \in \Pi} \sum_{v \in \mathscr{V}_{\pi}} \sum_{r=p_{\pi v}^{s_{0}}} \delta_{r a}, \quad a \in \mathscr{A} .
$$

Let $\mathscr{P}_{\pi v}$ be the set of restoration routes for VP $v$. For the VPI preassignment method, similar to $\mathrm{UV}(a)$, the number of reserved VPIs on arc $a$ can be calculated from

$\mathrm{RV}(a)=\sum_{\pi \in \Pi} \sum_{v \in \mathscr{V}_{\pi}} \sum_{r \in \mathscr{P} v} \delta_{r a}, \quad a \in \mathscr{A}$

As a network failure will only affect a portion of working VPs, for the non-preassignment method, the amount of VPIs reserved on each arc should be equal to the maximal number of restoration routes passing that arc under all possible failure cases. Given the affected working VP set $\mathscr{V}_{\pi}^{s}$ for a node pair $\pi \in \Pi$ and the restoration route $p_{\pi v}^{s}$ of each affected working VP in failure state $s$, we have

$\operatorname{RV}(a)=\max _{s \in \mathscr{P}_{-s_{0}}}\left\{\sum_{\pi \in \Pi} \sum_{v \in \mathscr{V}_{\pi}^{s}} \sum_{r=P_{\pi v}^{s}} \delta_{r a}\right\}, \quad a \in \mathscr{A}$.

Suppose that all working VPs take the shortest paths between origin and destination node pairs. The optimal restoration routes can be obtained from the IP solution described in Section 2. Fig. 4 compares the VPI redundancy of preassignment (p) and non-preassignment (np) methods for four different networks, where $\operatorname{Net}(11,17)$ and $\operatorname{Net}(20,42)$ are the networks described in Ref. [5] which have the same number of nodes as LATA_Net and US_Net, respectively. As expected, preassignment method need to reserve much more VPIs, especially when the SD restoration is used. For the non-preassignment method, the VPI redundancy is slightly smaller in the SI scheme than

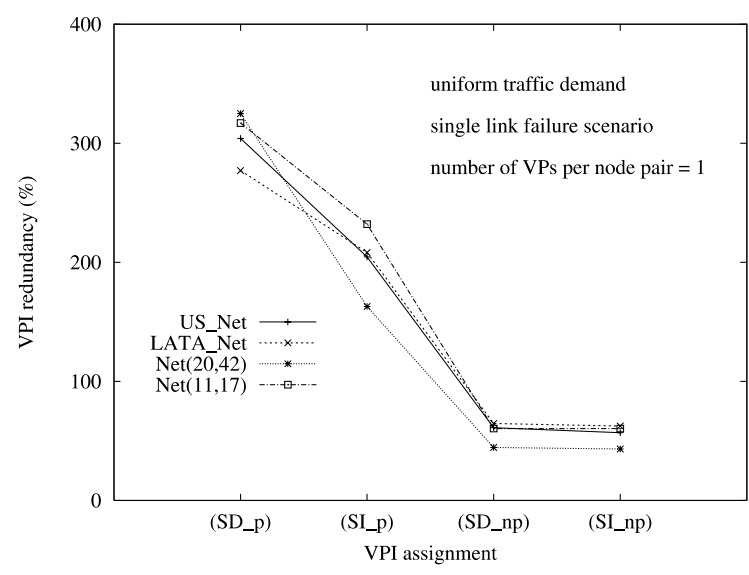

Fig. 4. Effect of assignment methods on VPI redundancy.

that in the SD scheme. In non-preassignment method, we also observe that the VPI redundancy is not very sensitive to $V$, the number of VPs of each node pair [12]. Although the uniform traffic demand is considered here, similar properties are also expected for non-uniform traffic demand.

\subsection{Node storage capacity}

Using the SD or SI scheme, a database need to be established in each node to store restoration information. The storage format for the SI scheme is shown in Fig. 5(a) where WVPI stands for working VPI and L_in denotes the incoming link of a switch. For the non-preassignment method, dummy VPIs are used as indicated by VPI*, which will be assigned unused VPIs only during the restoration process. The storage format for the SD scheme is similar to Fig. 5(a) except the origin node, which is given in Fig. 5(b).

(a) SI format:

$$
\begin{array}{rl|l|l|l|}
\text { Origin node: } & \text { L_in } & \text { WVPI_in } & \text { L_out } & \text { VPI*_out } \\
\text { Intermediate node: } & \text { L_in } & \text { VPI*_in } & \text { L_out } & \text { VPI*_out } \\
\cline { 2 - 5 } & & & \\
\text { Destination node: } & \text { L_in } & \text { VPI*_in } & \text { L_out } & \text { WVPI_out } \\
\cline { 2 - 4 }
\end{array}
$$

(b) SD format:

$$
\text { Origin node: } \begin{array}{|l|l|l|l|l|}
\text { Failure ID } & \text { L_in } & \text { WVPI_in } & \text { L_out } & \text { VPI*_out } \\
\hline
\end{array}
$$

Fig. 5. Node storage formats for SI and SD schemes. 
Table 7

Ratio of database entries of SD and SI schemes

\begin{tabular}{lllll}
\hline Network & LATA_Net & Net $(11,17)$ & Net $(20,42)$ & US_Net \\
\hline$E_{\mathrm{SD}} / E_{\mathrm{SI}}$ & 1.37 & 1.40 & 2.00 & 1.52 \\
\hline
\end{tabular}

Denote by $E_{\mathrm{SI}}$ and $E_{\mathrm{SD}}$ the average number of entries of the database when using the SI and SD schemes, respectively. We get

$E_{\mathrm{SI}}=\frac{1}{|\mathscr{N}|} \sum_{\pi \in \Pi} \sum_{v \in \mathscr{V}_{\pi}} \sum_{r \in \mathscr{P}_{\pi v}} n(r)$

and

$E_{\mathrm{SD}}=\frac{1}{|\mathscr{N}|} \sum_{\pi \in \Pi} \sum_{v \in \mathscr{V}_{\pi}}\left(m\left(p_{\pi v}^{s_{0}}\right)+\sum_{r \in \mathscr{P} v}[n(r)-1]\right)$,

where $n(r)$ is the number of nodes traversed by the route $r$, and $m\left(p_{\pi v}^{s_{0}}\right)$ is the number of possible failures that will affect the route $p_{\pi v}^{s_{0}}$ of working VP $v$. For the origin node of route $p_{\pi v}^{s_{0}}$, it need $m\left(p_{\pi v}^{s_{0}}\right)$ entries. For single link failure scenario, $m\left(p_{\pi v}^{s_{0}}\right)=$ $n\left(p_{\pi v}^{s_{0}}\right)-1$. Note that the set $\mathscr{P}_{\pi v}$ contains only one element (route) in the SI restoration, while it may contain more than one element in the SD restoration.

The ratio of $E_{\mathrm{SD}}$ and $E_{\mathrm{SI}}$ is shown in Table 7 for the uniform traffic demand $(V=1)$ and under a single link failure scenario [5]. It indicates that the node storage capacity required by the SD scheme could be as much as 2 times or more as that required by the SI scheme.

\section{Restoration time}

The restoration time of an affected working VP is defined here as the elapsed time from the failure occurrence to the completion of the restoration process. It is the sum of the failure detection time, round trip time between VP termination nodes and the restoration cell delays in network nodes along the backup VP route. The first two times can be easily estimated since they are mainly determined by the propagation delay, while the node delay is difficult to estimate because it is a random variable which depends on other affected working VPs and their backup VP routes. Here we resort to com- puter simulations to investigate the dynamic behavior of the restoration processes after network failure occurs.

When a network failure is detected, each node (sender) which is responsible for restoring the affected working VPs will trigger a restoration process. The restoration process first searches for the pre-planned backup VP route in the node database for an affected working VP and then send the selfhealing OAM (operations, administration and maintenance) cell along the backup route. Each node on the backup route initiates a processing function after receiving the OAM cell and then forwards it to the next node after completion of the processing function. The processing function basically checks the bandwidth availability on the outgoing link and updates the VP routing table. Upon receiving an acknowledgment OAM cell from the destination node (chooser), the sender can then switch the traffic of the affected working VP to the backup VP and the restoration process for this working VP is completed.

Receiving and sending self-healing OAM cells in a network node are performed by the handling function which includes cell dropping and inserting. The dropping function drops arriving OAM cells from the transmission line and forwards them to the processing function. The inserting function inserts processed OAM cells to the transmission line. As dropping, processing and inserting functions are running concurrently, we model a network node as a system of three tandem queues depicted in Fig. 6, where $T_{\mathrm{d}}, T_{\mathrm{p}}$ and $T_{\mathrm{i}}$ denotes dropping time, processing time and inserting time, respectively. Buffers are required to resolve contentions of self-healing OAM cells from affected working VPs.

Let $\operatorname{AD}(s)$ be the restorable affected traffic demands in network failure state $s$, and $R D(s, t)$ be the restored traffic demands at elapsed time $t$ in failure state $s, s \in \mathscr{S}-s_{0}$. We use the aggregate

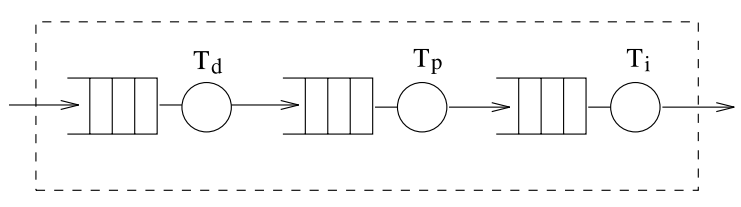

Fig. 6. A node model during restoration. 
restoration ratio at elapsed time $t$ to measure the restoration speed, which is defined as the ratio of total restored traffic at time $t$ to the total affected traffic in all possible failure cases, i.e.,

restoration_ratio $(t)=\frac{\sum_{s \in \mathscr{S}_{-s_{0}}} R D(s, t)}{\sum_{s \in \mathscr{S}_{-s_{0}}} A D(s)}$.

Denote by $T_{\mathrm{h}}$ the OAM cell handling time which is equal to the sum of dropping time $T_{\mathrm{d}}$ and inserting time $T_{\mathrm{i}}$,

$T_{\mathrm{h}}=T_{\mathrm{d}}+T_{\mathrm{i}}$.

It is assumed that $T_{\mathrm{d}}, T_{\mathrm{p}}$ and $T_{\mathrm{i}}$ are all constant in our simulation study and $T_{\mathrm{d}}=T_{\mathrm{i}}=T_{\mathrm{h}} / 2$. The handling time $T_{\mathrm{h}}$ is used as the basic time unit in the following numerical examples and let

$T_{\mathrm{r}}=T_{\mathrm{p}} / T_{\mathrm{h}}$.

Figs. 7 and 8 depicts the restoration ratio versus time in LATA_Net and US_Net (US_Net $(20,30)$ ), respectively, based on the optimal results obtained in Section $3(M=10)$ under one single link failure scenario. Only the SI restoration is shown here. Very similar results are also observed for the SD restoration [12]. In these two figures, the number of VPs per node pair is 5 and the self-healing OAM cell processing time $T_{\mathrm{p}}=0.25 T_{\mathrm{h}}$. For group VP restoration, all the 5 VPs of each node pair have the same working and backup paths, and the optimal results can be easily obtained by setting $\left|\mathscr{V}_{\pi}\right|=1$ in formulations $\mathrm{F} 1$ and F3. We assume

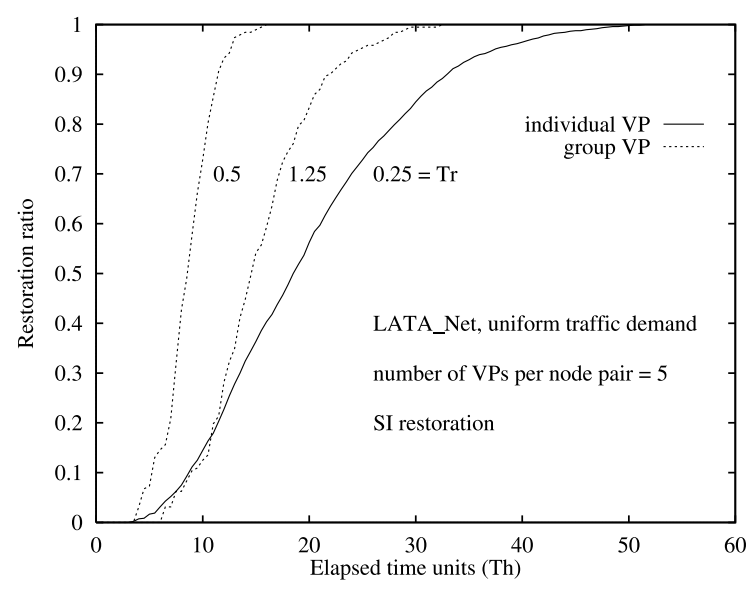

Fig. 7. Restoration ratio versus time in LATA_Net.

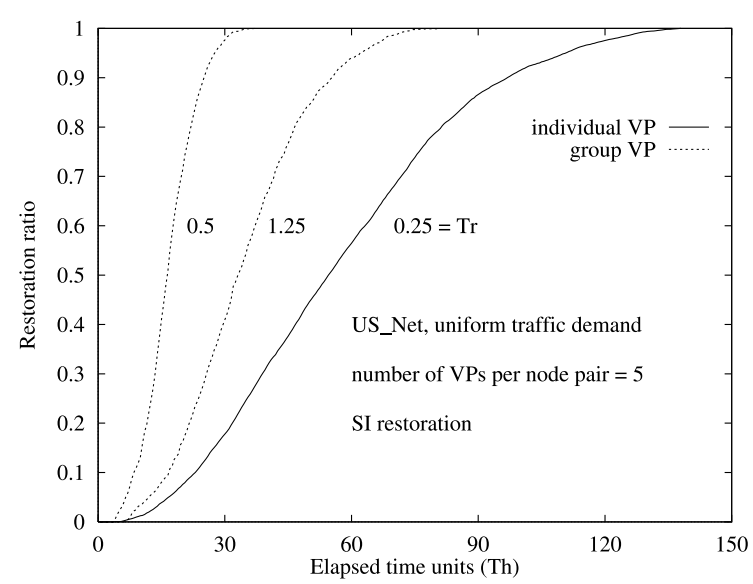

Fig. 8. Restoration ratio versus time in US_Net.

that in group VP restoration the cell processing time in group VP restoration is $2\left(T_{\mathrm{r}}=0.5\right)$ or 5 $\left(T_{\mathrm{r}}=1.25\right)$ times as that in the individual VP restoration. As one can see from Figs. 7 and 8, even if the cell processing time in group VP restoration is proportional to the number of VPs in the group, the restoration speed is still much faster. The reason is that using group VP restoration can substantially reduce the number of self-healing OAM cells generated upon network failure (4 times less in the above examples) and therefore ease the cell contentions in network nodes.

From the data provided in Ref. [13], $T_{\mathrm{d}} \approx$ $T_{\mathrm{i}} \approx 35 \mathrm{~ms}$ and $T_{\mathrm{p}} \approx 2-20 \mathrm{~ms}$, i.e., $T_{\mathrm{h}} \approx 70 \mathrm{~ms}$ and $T_{\mathrm{r}}=T_{\mathrm{p}} / T_{\mathrm{h}} \approx 0.03-0.29$. Fig. 9 further illustrates that if $T_{\mathrm{r}}$ is small, i.e., the cell processing time $T_{\mathrm{p}}$ is much less than the cell handling time $T_{\mathrm{h}}$, the advantage of group VP restoration is more obvious. The gap decreases as $T_{\mathrm{r}}$ gets large. When $T_{\mathrm{r}}$ is large enough (e.g., $T_{\mathrm{r}} \geqslant 1$ ) and the cell processing time is still proportional to the number of VPs in the group, then the group VP restoration may not have advantages because now the bottleneck is in cell processing rather than cell handling.

The impact of network size on restoration time is shown in Figs. 10 and 11. Networks with 15, 20 and 25 nodes are subnets of US_Net in Fig. 3. The network with 28 nodes are US_Net itself. The network with 11 nodes is $\operatorname{Net}(11,17)$ given in Ref. [5]. They are all sparse networks with connectivity in the range of 3.0-3.2. We see that the advantage 


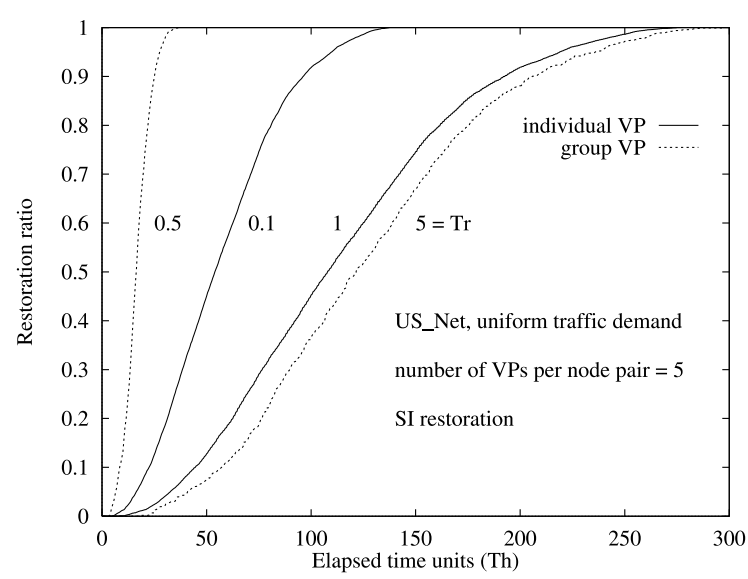

Fig. 9. Impact of $T_{\mathrm{r}}$ on the restoration ratio.

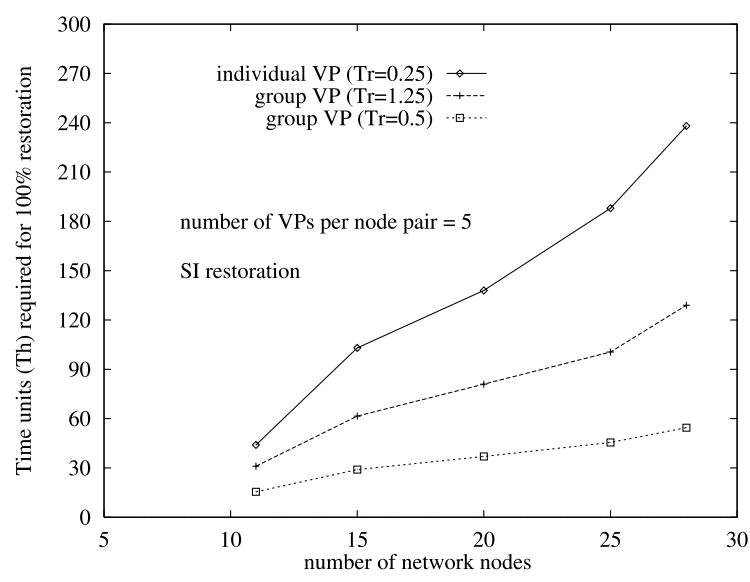

Fig. 10. Time for $100 \%$ restoration in different networks.

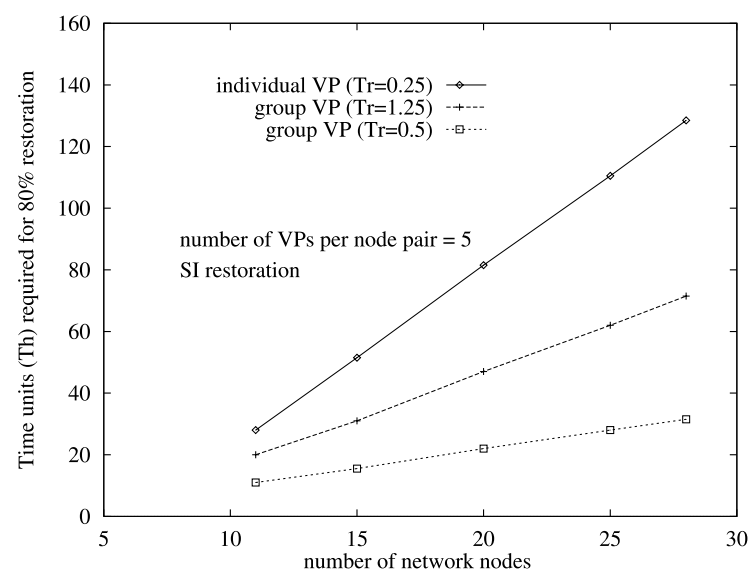

Fig. 11. Time for $80 \%$ restoration in different networks. of group VP restoration could be more significant in large networks. Interestingly, it appears that the time required for $80 \%$ restoration increases almost linearly with the network size.

\section{Concluding remarks}

In this paper, we have compared the preplanned SI and SD path restoration schemes in terms of SCR, restoration time, VPI redundancy and nodal storage requirement, based on optimal IP solutions. From the numerical results, it is found that SI path restoration appears to be better than the $\mathrm{SD}$ path restoration, at least in spare optimization, as the difference in SCR between SI and SD is only marginal. Further, for both SI and SD schemes, group VP restoration can greatly increase the restoration speed, while the penalty in SCR is not very significant (e.g., less than 5\%).

SCR depends on network topology, traffic demand and the optimization approach used, etc. Here we only examined a very limited set of network data. In cases where the difference in SCR between SI and SD cannot be ignored (e.g., in joint optimization) and the VPI non-preassignment method is used (or high VPI redundancy is tolerable), the SD scheme may be a good candidate. The VPI non-preassignment method can be used to solve the potential problem of storage of VPI resource. However, whether to use it or not will depend on how fast we can assign VPIs to backup routes.

\section{References}

[1] R. Kawamura, K.-I. Sato, I. Tokizawa, Self-healing ATM networks based on virtual path concept, IEEE J. Select. Areas Commun. 12 (1) (1994) 120-127.

[2] J. Anderson, B.T. Doshi, S. Dravida, P. Harshavardhana, Fast restoration of ATM networks, IEEE J. Select. Areas Commun. 12 (1) (1994) 128-138.

[3] R. Doverspike, B. Wilson, Comparison of capacity efficiency of DCS network restoration routing techniques, J. Network Syst. Mgmt. 2 (2) (1994) 95-123.

[4] R. Iraschko, M. MacGregor, W. Grover, Optimal capacity placement for path restoration in mesh survivable networks, in: Proceedings of ICC'96, 1996, pp. 1568-1574.

[5] Y. Xiong, L. Mason, Restoration strategies and spare capacity requirements in self-healing ATM networks, IEEE/ACM Trans. Networking 7 (1) (1999) 98-110. 
[6] Using the CPLEX Callable Library, Version 5.0, 1997, CPLEX Division, ILOG Inc., NV, USA.

[7] P. Veitch, D. Smith, I. Hawker, A comparison of preplanned routing techniques for virtual path restoration, in: D. Kouvatsos (Ed.), Performance Modeling and Evaluation of ATM Networks, vol. 2, Chapman and Hall, New York, 1996.

[8] K. Murakami, H. Kim, Joint optimization of capacity and flow assignment for self-healing ATM networks, in: Proceedings of IEEE ICC'95, 1995, pp. 216-220.

[9] M. Herzberg, S.J. Bye, A. Utano, The hop-limit approach for spare-capacity assignment in survivable networks, IEEE/ACM Trans. Networking 3 (6) (1995) 775-784.

[10] M. Herzberg, A. Utano, Optimal assignment of spare capacity to backup VPs in survivable ATM networks, in: Proceedings of NETWORKS'96, Sydney, 1996, pp. 665-760.

[11] K. Murakami, H. Kim, Comparative study on restoration schemes of survivable ATM networks, in: Proceedings of IEEE INFOCOM'97, Kobe, 1997, pp. 345-352.

[12] Y. Xiong, L. Mason, On state-independent and statedependent path restoration in self-healing networks, in: Proceedings of IEEE ICC'98, Atlanta, 7-11 June 1998, pp. 1114-1118.

[13] H. Saito, M. Slominski, M. Yoshida, An improved guided restoration algorithm for ATM crossconnect networks, in: Proceedings of IEEE Network Operations and Management Symposium, Kyoto, 1996, pp. 225-234.

[14] S. Han, K. Shin, Fast restoration of real-time communication service from component failure in multi-hop networks, in: Proceedings of ACM SIGCOMM'97, 1997, pp. 77-88.

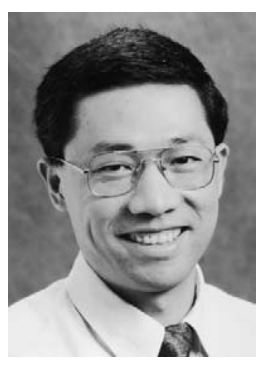

Yijun Xiong received the $\mathrm{Ph} . \mathrm{D}$. degree in Electrical Engineering from the University of Ghent, Belgium, in 1994. $\mathrm{He}$ is now a member of Technical Staff at Mahi Networks Inc., Petaluma, CA. From August 1998 to December 2000, he was a senior research scientist at Alcatel Corporate Research Center in Richardson, Texas. From October 1996 to August 1998, he was an Assistant Professor of Electrical and Computer Engineering at Louisiana State University, Baton Rouge, LA.
His current research interests include optical packet/burst networks, switch architectures, network survivability, traffic modeling and network performance analysis.

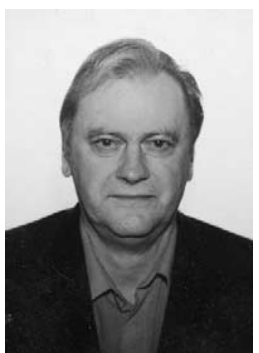

Lorne G. Mason is a Visiting Professor in the Department of EEE (ICIS), Nanyang Technological University and Professeur Honouraire at INRS-Telecommunications, University of Quebec. Dr. Mason obtained the B.Sc., Post Graduate Diploma, and Ph.D. degrees in Mechanical Engineering from the University of Saskatchewan, Canada, in 1963, 1969 and 1973, respectively. He was with Bristol Aerojet in Winnipeg, Manitoba, from 1963 to 1965 involved in the design of the Black Brant rockets. He joined the British Columbia Telephone Co. as a Traffic Engineer in 1966 and again in 1972 as a Consultant for planning digital networks. In 1973 he served as a Consultant to Yale University, where he, in collaboration with Professor K.S. Narendra, pioneered the use of learning automata for adaptive routing in telecommunication networks. Between 1974 and 1977, he was with Bell-Northern Research where he developed planning tools and methods for digital network evolution and state-dependent routing. Since1977 he has been with INRS-Telecommunications where he successively held the titles of invited, associate, full professor and recently professeur honouraire. During this period he conducted research and taught post-graduate students in telecommunications. His primary research interests are the application of control theory and operations research methods to telecommunication network design, control, and management. He has held numerous industrial research contracts and strategic grants from the Natural Sciences and Engineering Research Council of Canada, in the area of broadband network design and analysis and has over 80 publications on the subject in leading conferences and journals. Professor Mason was a participant in the Network of Centers of Excellence program sponsored by the Canadian Government, where he was project leader in the area of broadband network control. He was Co-recipient of the 1993 STENTOR Award for collaborative research in telecommunications for his contributions to statedependent routing. He organized and chaired the joint IFIP/ IEEE conference, Broadband Communications '96, and was editor of the proceedings published in book form by Chapman and Hall. He was Technical Co-chairman for the IEEE ATM'98 workshop, and technical program committee member for several international conferences in the broadband area. He is a CIPS (Canadian Institute for Information Processing) member and Canadian representative to IFIP TC6 committee and a senior member of IEEE. 